\title{
Impact of Mutations on K-Ras-p120GAP Interaction
}

\author{
Chunxia Gao, Leif A. Eriksson* \\ Department of Chemistry and Molecular Biology, University of Gothenburg, Göteborg, Sweden \\ Email: *1eif.eriksson@chem.gu.se
}

Received January 28, 2013; revised February 28, 2013; accepted March 8, 2013

Copyright (C) 2013 Chunxia Gao, Leif A. Eriksson. This is an open access article distributed under the Creative Commons Attribution License, which permits unrestricted use, distribution, and reproduction in any medium, provided the original work is properly cited.

\begin{abstract}
The K-Ras protein plays a key role in the signal transduction cascade. Certain mutations in K-Ras lead to a permanent "on" state which results in tumorigenesis due to failed interaction with the GTPase activating protein (GAP). In this study, we examined the mutations E31N, D33N and D38N of K-Ras coupled and decoupled to wildtype GAP-334 and mutation K935N of GAP-334 coupled and decoupled to wildtype K-Ras, to illustrate the potential mechanism by which these mutants affect the interaction between the two proteins. We identify Tyr32 in the Ras Switch I region as a critical residue that acts as a gate to the GTP binding site and which needs to be "open" during Ras coupling with GAP to allow for insertion of GAP residue Arg789. This residue plays a vital role in stabilizing the transition state during GTP hydrolysis. The different mutations studied herein caused a reduced binding affinity, and the fluctuation of the Tyr32 side chain might hinder the insertion of Arg789. This may in turn be the cause of decreased GTP hydrolysis, and permanent "on" state of K-Ras, observed for these mutants.
\end{abstract}

Keywords: Ras Protein; GTPase Activating Protein; Molecular Dynamics Simulations; In Silico Mutation Studies; Cancer

\section{Introduction}

The Ras proteins, H-, N- and K-Ras, are low molecular weight GTPases that play important roles in signal transduction of cell proliferation and/or differentiation [1]. In the active state, Ras binds guanosine triphosphate (GTP), and inactivation occurs by conversion of GTP to guanosine diphosphate (GDP). The rate of this reaction is crucial for the corresponding timing of the signal transduction, and requires the Ras protein to hydrolyze GTP to GDP in order to complete the normal activation of downstream effector proteins. The intrinsic low GTPase activity of Ras can be dramatically increased by binding to the GTPase activating protein (GAP). However, many oncogenic mutants of Ras escape regulation by GAP, which leads to Ras are always in its active state. The longer Ras remains in the active GTP-bound state, the longer it will transmit and also amplify certain signals, eventually leading to uncontrolled multiplication of cancer cells [2-4].

The vast majority of Ras mutations found in human disease occur in K-Ras, with mutations in H-Ras and $\mathrm{N}$-Ras being quite rare $[5,6]$. Interestingly, the primary structures of these three Ras proteins are almost identical; in particular the amino acid sequences of H-Ras and

\footnotetext{
${ }^{*}$ Corresponding author.
}

K-Ras are identical until residue 95 , and with only two differences up to residue 120 (see supporting Figure S1). Several mammalian Ras specific GAPs of various sizes and molecular architectures have been described to date. They all have high sequence similarity in their catalytic GAP domains, which are segments of 250 - 350 amino acid residues. The most prominent and best studied GAPs are p120GAP and neurofibromin [7]. The catalytic domains of neurofibromin (residues 1198-1530, NF1-333) and p120GAP (residues 714-1047, GAP-334), interact with all three Ras isoforms without preference.

A likely catalytic mechanism for hydrolysis of Rasbound GTP by GAP has been illustrated through the Xray structure of an H-Ras-GAP complex in which H-Ras was bound to GDP and $\mathrm{AlF}_{3}$, where $\mathrm{AlF}_{3}$ resembles the cleaved $\gamma$-phosphate group of GTP [8]. GAP-334 contains eight helices (denoted $\alpha 1 \mathrm{c}-\alpha 8 \mathrm{c}$ ) separated by six loops (denoted L1c - L6c). Of these, the elements that contact with Ras are $\alpha 6 \mathrm{c}, \alpha 7 \mathrm{c}, \mathrm{L} 1 \mathrm{c}$, and L6c. L1c is called "finger loop" because it contains residues that enter into the GTP binding site of Ras; in particular Arg789, which interacts with Gly12, Gln61, and the fluoride atoms of $\mathrm{AlF}_{3}$. This residue has therefore been postulated to neutralize the developing negative charge on the GTP phosphate oxygen atoms during phosphoryl cleavage [9- 
11]. GAP furthermore interacts with Ras Switch I and Switch II regions (containing amino acids 30 - 40, and 60 - 70, respectively), and stabilizes these two regions during the hydrolytic process (Figure 1). Different from previously identified mutation hot spot codons 12,13 and 61 in tumors [12], some point mutations in the K-Ras Switch I region, including E31N, D33N and D38N, have recently been detected in cervical cancer and adrenal tumors [13-16]. In addition, Lys1423 in NF1-333 mutated to Glu or Gln in neurofibromas and solid tumors, have claimed to completely impair the GTPase stimulating activity of NF1-333 [17]. The corresponding residue in GAP-334, Lys935, is involved neither in binding nor catalysis; instead the side chain of Lys935 is located near Ile36, Glu37 and Asp38 of Switch I (Figure 2). Hence, either Ras mutated in the Switch I region, or transformed GAP with a mutated Lys residue spatially close to the Switch I region, seem crucial in oncogenicity. Judging from the positions of these point mutations, they cannot be directly involved in the catalytic reaction in the same way as the hot spot codons $(12,13,61)$ which are in direct contact with the phosphoryl group of GTP. To explore how these mutants hinder the GTPase function of GAP, we have used molecular dynamics (MD) simulations to compute the effects of each mutant on the interaction between GAP-334 and K-Ras. Our investigations reveal a critical residue Tyr32 in the Ras Switch I region, which acts as a gate to access GTP and appears to be "open" when Ras couples with GAP for insertion of Arg789. The different mutations studied herein seemingly cause an incomplete "open" state of Tyr32 and lead to failed insertion of Arg789 critical for GTP hydrolysis.

\section{Materials and Methods}

\subsection{Materials and Programmes}

The X-ray crystal structure of H-Ras bound to GAP-334 (PDB entry: $1 W Q 1$ ) [8], K-Ras with bound GTP analogue GppNHp (3GFT), and the GAP related domain of neurofibromin (1NF1) [18] were chosen as the initial structures.

All in silico mutations, structure superpositions and protonation state determinations of the residues were performed using the Molecular Operating Environment (MOE) 2009.10 program [19]. For MD simulations and energy minimization, the GROMACS software [20] (Version 4.0.4) together with the Amber 99 force field [21] was used.

\subsection{Preparation of the Systems}

MD simulations were performed on in silico mutated proteins in coupled and uncoupled forms to see how the

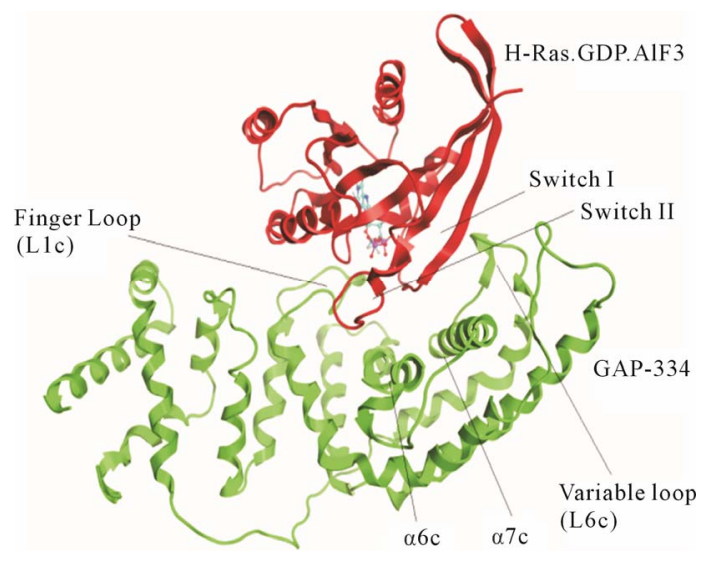

Figure 1. The complex between GAP-334 and H-Ras. The GAP-334 ribbon is colored green, H-Ras colored red. The contacting region of each protein is indicated.

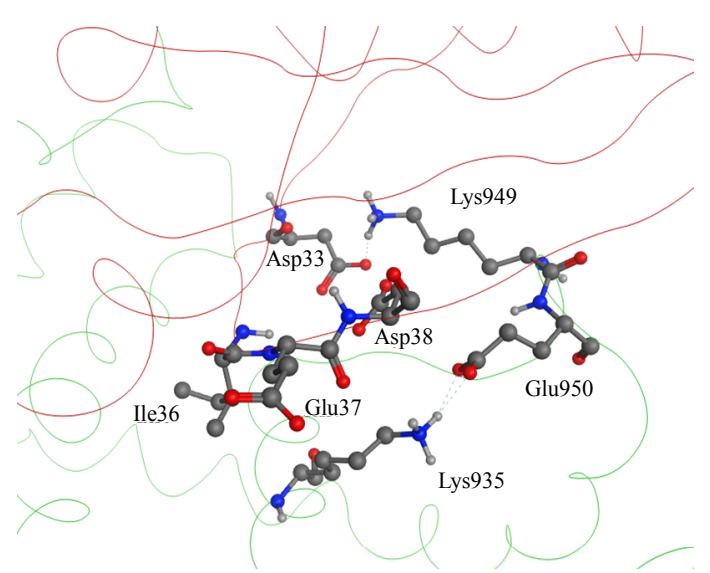

(a)

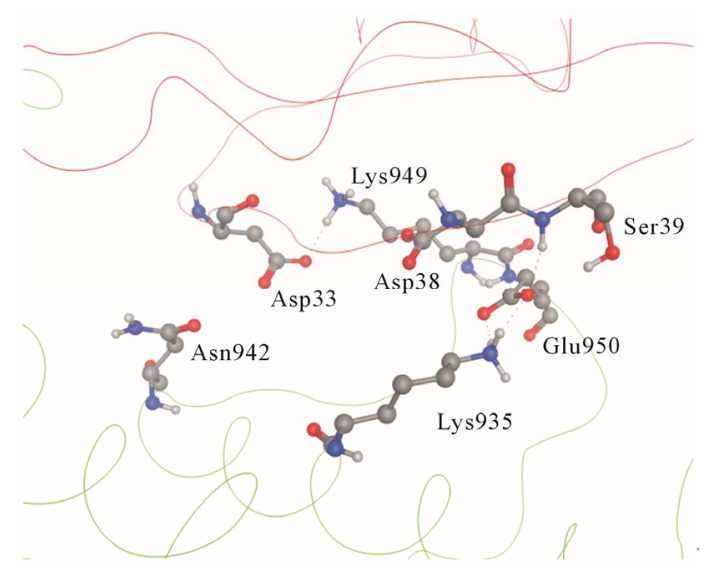

(b)

Figure 2. (a) Details of the interaction of H-Ras with GAP334. Switch I residues Asp33, Glu37 and Asp38 (backbone ribbon colored red) and GAP-334 residues Lys935, Lys949 and Glu950 (backbone ribbon green); (b) The Switch I region is fixated by Asn942, Lys949 and Glu950 of GAP-334. Asn942 forms a hydrogen bond with Asp33, Lys949 makes direct contact with Asp33 and water mediated polar contact with Asp38, and the carboxylate group of Glu950 interacts with the main chain amide group of Ser39. 
mutated residues affect the protein conformations in the different forms.

\subsubsection{Uncoupled Proteins}

Using the crystal structure of human K-Ras in complex with the GTP analogue GppNHp (3GFT), we transformed GppNHp to GTP by substituting the $\mathrm{N}$ to a $\mathrm{P}$ atom, with the bond lengths and the charge of the atom updated synchronously. Based on this $\mathrm{Mg}^{2+}$.K-Ras.GTP structure, MD simulations were carried out (parameters described below), followed by energy minimization of the structure from the last snapshot of the MD trajectories. In order to investigate if the K-Ras Switch I mutants have an effect on the protein conformation, in silico mutations E31N, D33N and D38N of the optimised K-Ras structure were performed, followed by MD simulations. For GAP, MD simulations were performed on both wildtype and mutant (K1423E) NF1-333 (crystal structure $1 N F 1$ ).

\subsubsection{Coupled Proteins}

The initial X-ray structure $1 W Q 1$ contains the substrate GDP-AlF $\mathrm{Al}_{3}$ bound to $\mathrm{H}-\mathrm{Ras}$, where the $\mathrm{AlF}_{3}$ molecule is thought to "mimic" the $\gamma$-phosphate moiety of GTP. In place of the $\mathrm{AlF}_{3}$ molecule, we thus generated the $\gamma$ phosphate group bound to GDP in the active site of $\mathrm{H}$ Ras. 10 different amino acids differ between H-Ras and K-Ras up to codon 95; we thus in silico mutated these ten amino acids, Q95H, D107E, A121P, A122S, E126D, S127T, R128K, Y141F, E153D and Q165K, to transform $\mathrm{H}-\mathrm{Ras}$ to K-Ras (see supporting Figure S1). We then performed MD simulation (parameters described below), followed by energy minimization on the new complex of $\mathrm{Mg}^{2+}$.K-Ras.GTP.GAP-334 from the last snapshot of the MD trajectories. Based on the energy optimized $\mathrm{Mg}^{2+}$. K-Ras.GTP.GAP-334 complex structure, another four separate in silico mutations were performed followed by MD simulations as outlined above. The single mutant complexes include E31N, D33N and D38N of K-Ras, and $\mathrm{K} 935 \mathrm{~N}$ of GAP-334, respectively, with the aim to explore the effects of these frequent mutants on complex stabilities and interactions.

\subsection{Molecular Dynamics Simulations}

The GROMACS software and Amber 99 force field were used for all the MD simulations. The parameters for GTP were obtained from Meagher et al. [22] (atomic charges given in Supporting Information). The structures were solvated in periodic boxes with a buffer distance of 10.0 $\AA$. A number of $\mathrm{Na}^{+}$and $\mathrm{Cl}^{-}$ions were added to satisfy the electro neutrality condition and to give a salt concentration of $0.1 \mathrm{~mol} / \mathrm{liter}$, using the genion module in GROMACS. The obtained systems ( $\sim 54,500$ atoms in the complex system, $\sim 19,000$ atoms in uncoupled K-Ras, and $~ 50,600$ in uncoupled NF1-333 GAP) were energy minimized by steepest descent (200 steps) to remove close contacts. Position restrained simulations ( 2 ns duration, 1.0 fs time step, NPT ensemble, $\mathrm{T}=298 \mathrm{~K}, \mathrm{P}=1$ bar) were first performed, to enable the water molecules to attain more favorable positions. Particle-mesh Ewald (PME) [23,24] summation was used for long-range electrostatics. A $12 \AA$ cutoff was used for both Coulomb and Lennard-Jones interactions. The temperature and pressure was controlled through the Berendsen coupling algorithm [25], with the time constants 0.1 ps for temperature and $1.0 \mathrm{ps}$ for pressure coupling. All bond lengths were constrained using the LINCS algorithm [26]. During the production MD simulations (30 ns duration, $1.0 \mathrm{fs}$ time step, NPT ensemble, $\mathrm{T}=298 \mathrm{~K}, \mathrm{P}=1 \mathrm{bar}$ ), the temperature was controlled using the Nose-Hoover thermostat [27] with a time constant $0.1 \mathrm{ps}$, and the pressure was controlled using the Parrinello-Rahman barostat [28], with a time constant 1.0 ps. The remaining parameters were the same as in the position restrained simulations.

\subsection{Interaction Energy Calculations}

The interaction energy (IE) is defined as the potential energy difference between the K-Ras.GTP.GAP complex (K-G) and the individual K-Ras. GTP (K) and GAP (G) systems, Equation (1).

$$
\mathrm{IE}=\mathrm{E}_{\mathrm{K}-\mathrm{G}}-\left(\mathrm{E}_{\mathrm{K}}+\mathrm{E}_{\mathrm{G}}\right)
$$

In order to eliminate effects of the kinetic energy, geometry optimizations were performed with the Amber 99 force field, on the 10 snapshots from the last $1 \mathrm{~ns}$ MD trajectories of each system (including solvent molecules and $\mathrm{Na}^{+}, \mathrm{Cl}^{-}$ions). The interaction energy calculations were then performed on the optimized geometries of the various K-Ras.GTP.GAP complexes with all water molecules and ions removed.

\section{Results and Discussion}

\subsection{Overall Dynamic Behaviour of Unbound Wildtype and Mutated K-Ras and NF1-333 GAP}

Simulations of the unbound wildtype and mutated K-Ras and NF1-333 GAP were performed to investigate if the mutations cawuse any severe structural differences before the two proteins bind together.

In the wildtype K-Ras protein, the side chains of residues E31, D33 and D38 are not interacting with any other surrounding residues; instead they all stretch out away from the GTP binding site (supplementary Figure S1(b)). After MD simulations, the mutated residues E31N, D33N and D38N keep the same position as in the wildtype. The RMSDs of the backbone structures were 
calculated for all simulations. The RMSD values stay fairly constant around $2 \AA$, and the mutants do not differentiate much from wildtype (Figure 3(a)). The backbone root-mean-square fluctuations (RMSFs) were also calculated since they can give an indication of backbone mobility. The backbone RMSFs fluctuate in a very similar manner for both wildtype and mutant proteins. For the Switch I region (residues 30 - 40), the RMSF values are very small, indicating that the region is stable (Figure 3(b)). Moreover, the average GTP to protein H-bond number from the last $1 \mathrm{~ns}$ of simulation in wildtype and mutants are the same (Table 1). We can thus conclude that these Switch I mutants in K-Ras do not influence the protein conformation itself, and do not interfere in GTP binding.

In wildtype NF1-333 GAP, K1423 is located on helix $\alpha 7 \mathrm{c}$ from which it protrudes into the surface groove to form a salt bridge with E1437. In the mutated K1423E structure, this salt bridge is broken. The E1423 carboxylic side chain keeps the same position as wildtype K1423, but the electrostatic charge repels E1437 and the surface groove becomes slightly less defined albeit retaining the same orientation (see supporting Figure S2). The fluctuations of backbone RMSD show that the conformation of mutant K1423E is more unstable compared to the wildtype NF1-333 GAP. For the wildtype system, it reaches to stabilization after $10 \mathrm{~ns}$ and retains a RMSD value of $4.5 \AA$; for the mutant, it fluctuates in the beginning, and stabilizes first after $15 \mathrm{~ns}$, whereafter it retains a relatively higher RMSD value of $5 \AA$ (Figure 3(c)).

In conclusion, mutations in the single protein K-Ras or NF1-333 GAP do not induce severe structural changes. The two proteins should in principle still be able to couple to each other despite these point mutations.

\subsection{Effects of Mutations E31N, D33N and D38N of K-Ras, and K935E of GAP, on Ras-GAP Interaction}

Since the GTPase activity of Ras proteins is stimulated by GAP, it has been proposed that certain mutations either in Ras or GAP would cause a deficiency in GTPase activity resulting in uncontrollable cell proliferation. The

Table 1. Number of hydrogen bonds for K-Ras and GTP in different systems, including errors.

\begin{tabular}{ccc}
\hline System & Uncoupled & Coupled \\
\hline Wildtype & $13.13 \pm 0.13$ & $16.64 \pm 0.15$ \\
E31N & $13.29 \pm 0.13$ & $16.35 \pm 0.14$ \\
D33N & $13.01 \pm 0.14$ & $16.78 \pm 0.14$ \\
D38N & $13.09 \pm 0.13$ & $16.77 \pm 0.17$ \\
K935E & - & $16.87 \pm 0.15$ \\
\hline
\end{tabular}

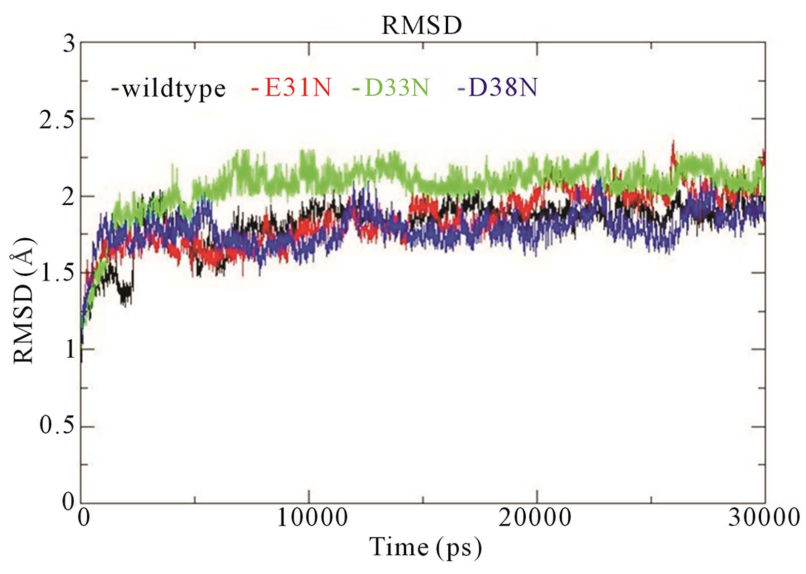

(a)

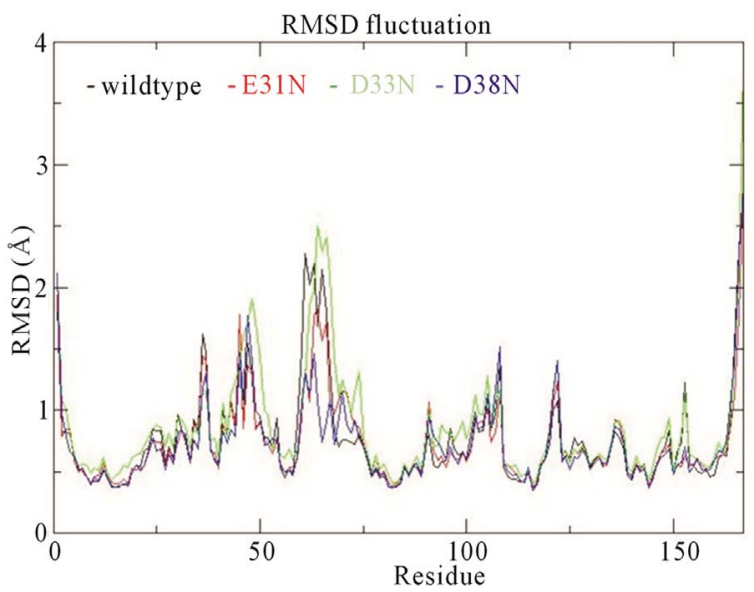

(b)

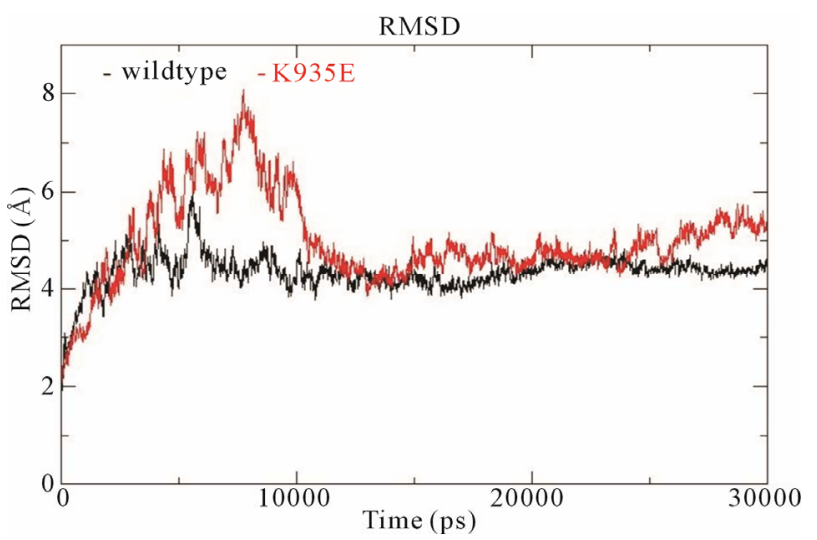

(c)

Figure 3. (a) Trajectories of the overall backbone RMSDs (in Å) of uncoupled K-Ras of the wildtype and mutant structures; (b) Backbone RMSF (in Å) of uncoupled K-Ras of the wildtype and mutant structures; (c) Trajectories of the overall backbone RMSDs (in $\AA$ ) of uncoupled NF1-333 GAP of the wildtype and mutant structures. All data with respect to the corresponding starting structures over $30 \mathrm{~ns}$ MD simulation.

mutation hot spots located on codons 12, 13 and 61 of Ras are well established and have been widely studied. 
However, the mechanisms behind why codons 31,33 and 38 of Ras, and codon 935 on GAP-334 (1423 on NF-333) also result in tumorigenesis are still largely unknown. To this end we performed $30 \mathrm{~ns}$ MD simulations of the different mutanted complex systems to predict the effects of mutations on protein structure and protein-protein interactions.

\subsubsection{Root-Mean-Square Deviations (RMSD)}

The RMSD trajectories of the backbone structures of mutant K-Ras or GAP-334 in the complexes display a different behaviour to the wildtype system (Figure 4). In the wildtype complex, both K-Ras and GAP-334 stabilize after $\sim 3 \mathrm{~ns}$; K-Ras RMSD varies between $1.0-1.25$ $\AA$ after stabilization, whereas that of GAP-334 varies between 1.75 and $2.25 \AA$. In the E31N system, K-Ras stabilizes rapidly and attains an RMSD value from 0.8 to $1.2 \AA$, whereas GAP-334 gradually drifts and stabilize first at around $\sim 20 \mathrm{~ns}$, from where it fluctuates between 2 to $3 \AA$. In the $\mathrm{D} 33 \mathrm{~N}$ system, K-Ras again evolves rapidly and stabilize at $\sim 1 \mathrm{~ns}$ from which it attains values of 0.8 $\sim 1 \AA$, whereas GAP-334 does not stabilize until after $\sim 12 \mathrm{~ns}$, and then attains values between $1.8-2 \AA$. For D38N, both K-Ras and GAP-334 reaches stabilization after $\sim 8 \mathrm{~ns}$, with K-Ras attaining values of $0.8-1 \AA$ and

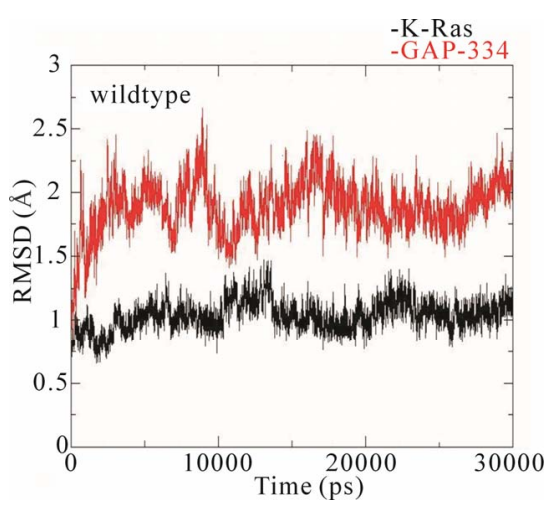

(a)

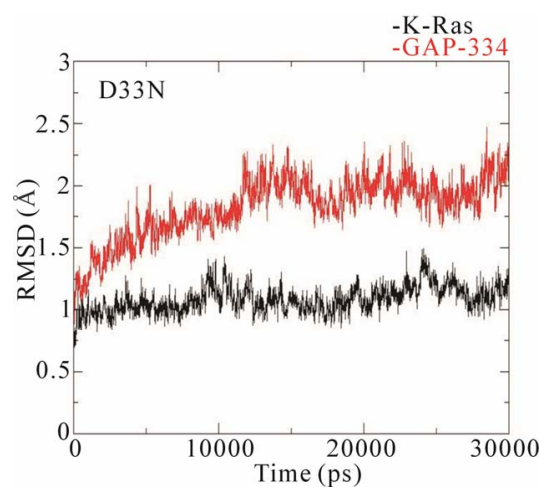

(c)
GAP-334 1.25 - 1.75 A. In K935E finally, K-Ras evolves rapidly to $1.5 \AA$ within the first $5 \mathrm{~ns}$, whereafter it drops to values from 0.8 to $1 \AA$, whereas the mutated GAP-334 gradually drifts to stabilize at around $\sim 20 \mathrm{~ns}$ and then fluctuates between 2 - $2.5 \AA$ (Figure 4).

It can be concluded that the RMSD trajectories of KRas do not differ significantly in any of the systems.

\subsubsection{Interaction Changes Caused by the Mutations} The RMSD trajectories of GAP-334 in the wildtype, D33N, and D38N systems are also quite similar, whereas in E31N and K935E, the RMSD trajectories of GAP-334 do not stabilize until after $20 \mathrm{~ns}$, and at higher values, which reflects larger structural deviations occurring in GAP-334 in these two systems.

\subsubsection{Interaction Energies}

Table 2 lists interaction energies between K-Ras.GTP and GAP of the wildtype and mutant systems. Due to themutations, the overall K-Ras.GTP.GAP interaction for the mutant complex becomes weaker compared to the wildtype system. The K935E mutation causes a significant reduction in interaction energy. The K-Ras mutant systems cause less interaction energy difference; 36 $\mathrm{kcal} / \mathrm{mol}$ in $\mathrm{E} 31 \mathrm{~N}, 44 \mathrm{kcal} / \mathrm{mol}$ in $\mathrm{D} 33 \mathrm{~N}$, and $13 \mathrm{kcal} / \mathrm{mol}$

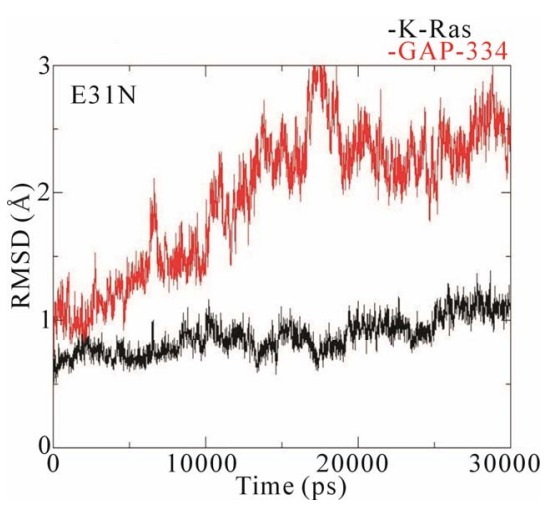

(b)

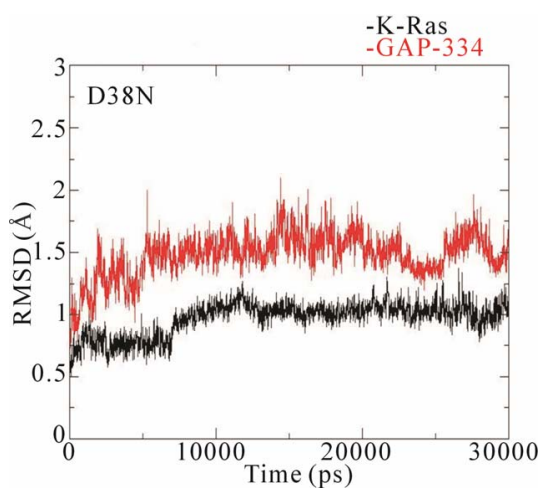

(d)

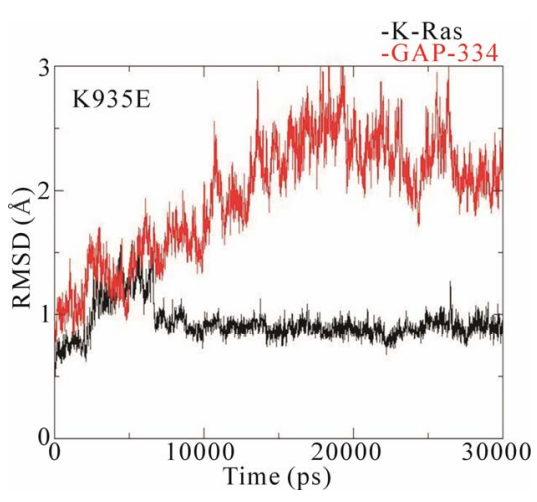

(e)

Figure 4. Trajectories of the overall backbone RMSDs (in $\AA$ ) of K-Ras (black) and GAP-334 (red) in the wildtype and mutant complexes with respect to the starting structure over $30 \mathrm{~ns}$ MD simulation. 
Table 2. Interaction energies of wildtype and mutant systems, including errors.

\begin{tabular}{cc}
\hline System & Interaction energy $(\mathrm{kcal} / \mathrm{mol})$ \\
\hline Wildtype & $-861.8 \pm 12.7$ \\
E31N & $-825.9 \pm 9.5$ \\
D33N & $-817.1 \pm 15.1$ \\
D38N & $-849.0 \pm 11.0$ \\
K935E & $-707.9 \pm 16.1$ \\
\hline
\end{tabular}

in $\mathrm{D} 38 \mathrm{~N}$.

In the wildtype, the K-Ras Switch I region is fixated by Asn942, Lys949 and Glu950 of GAP-334. Asn942 forms a hydrogen bond with Asp33, Lys949 makes direct contact with Asp33 and water mediated polar contact with Asp38, and the carboxylate group of Glu950 interacts with the main chain amide group of Ser39 (Figure 2(b)).

In the E31N system, the side chain of Asn31 orients away from the binding interface and towards to the core of the Switch I region.

In the D33N system, the mutated residue Asn33 hydrogen bonding to Asn942 and Lys949 of GAP-334 is disrupted; instead, Lys949 orients completely towards Asp38 and forms hydrogen bond with it and Glu950, with a concomitant increase in distance to Asn33.

For the D38N system, the mutated residue loses its hydrogen bond to Lys949; however, Lys949 retains its hydrogen bond with Asp33.

$\mathrm{K} 935 \mathrm{E}$, finally, is a highly unfavorable mutation, which gives a loss in salt bridge to Glu950. Due to the disrupted salt bridge, Lys949 and Glu950 fluctuate significantly, whereby Lys949 loses its interaction with Asp33, and Glu950 with Ser39. Asn942 furthermore also loses its contact with Asp33.

Based on the above findings, we conclude that the complementary attractive electrostatic interaction plays an important role for the protein-protein interaction and recognition. Mutation of negatively charged residues located in the Switch I region, or positively charged residues of GAP spatially close to the Switch I region, affect the electrostatic interaction of the two proteins and reduce their affinity to each other. Moreover, the unaffected average number of $\mathrm{H}$-bonds of GTP to the proteins demonstrates that the mutations do not interrupt the GTP binding (Table 1).

\subsubsection{Role of the Tyr32 Gate}

In order to better understand the characteristics of the protein structures, we superposed the overall structures of the H-Ras.GAP-334 complex on those of the isolated molecules, using free GAP-334 and H-Ras for compareson. This reveals that the side chain phenolic ring of
Tyr32 from Switch I deviates significantly when the system is in complex with GAP and when it is not. In the unbound form, the phenolic ring of Tyr32 shields the $\beta$ and $\gamma$ phosphate groups of GTP, whereas in the GAP coupled form, the side chain is moved aside such that the $\beta$ and $\gamma$ phosphates are exposed to Arg789 of GAP which thereby can enter into the active site and exert its catalytic role (see Figure $\mathbf{S 3}$ ).

In a simulation using the $\mathrm{K}$-Ras protein isolated from the Ras-GAP complex, the Tyr32 gate rapidly moved back to contact with the $\gamma$ phosphate group of GTP as in the unbound K-Ras structure (see Figures S4). The reorientation of side chain Tyr32 has also been noted by simulation of active GTP-bound and inactive GDPbound Ras [29,30]. We hence propose that Tyr32 acts as a gate governing the accessibility to the GTP phosphate group. The mutations in the Switch I region might cause fluctuations of the Tyr32 gate such that it does not provide enough space for insertion of Arg789 and inhibits GAP to excert its catalytic role.

We thus analysed the distance between the atoms of the side chain phenol ring of Tyr32 and the atoms in the guanidinium group of Arg789 in the different MD simulations. In the wildtype system, the distance fluctuates around $7 \AA$ most of the time during the simulation. This distance is thus characteristic of Tyr32 in its open conformation with Arg789 fully inserted into the active site. In the E31N system, the distance fluctuates around $6.5 \AA$ during the initial $12 \mathrm{~ns}$, where after the distance suddenly drops to $4.5 \AA$; in the D33N system, the distance fluctuates around $6 \AA$ for the initial $15 \mathrm{~ns}$ and then drops to $4 \AA$. For D38N, the distance is reduced to $5 \AA$ during the initial $5 \mathrm{~ns}$, and then fluctuates around $6 \AA$ most of the time; in the $\mathrm{K} 935 \mathrm{~N}$ system, finally, the distance fluctuates around $6.5 \AA$ for the first $10 \mathrm{~ns}$, and then drops to $5 \AA$ for the rest of the simulation (Figure 5).

Although it is not evident from these data that the side chain phenol ring of Tyr32 interferes with the guanidineium group of Arg789 since the simulations were started from an already interacting complex, the mutations clearly bring about changes that lower the distance between them; i.e., that the side chain phenol ring of Tyr32 is moving towards the guanidinium group of Arg789. Since the MD simulations are started from the systems with mutations introduced a posteriori to protein-protein complex formation, the Tyr32 gate is already "fixed" to some degree in the initially open state and is not able to move freely. The data obtained is however sufficient to conclude that in the initial interaction between Ras and GAP, the mutations cause an incomplete opening of the Tyr32 gate, which in turn hinders the insertion of Arg789 required for the catalysis.

\section{Conclusion}

We have performed computational studies on wildtype 


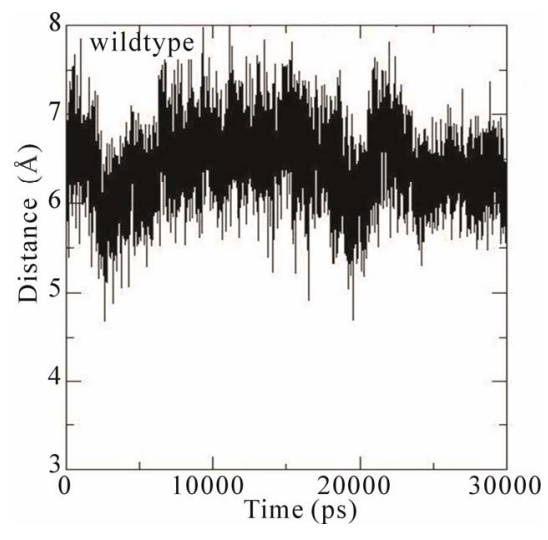

(a)

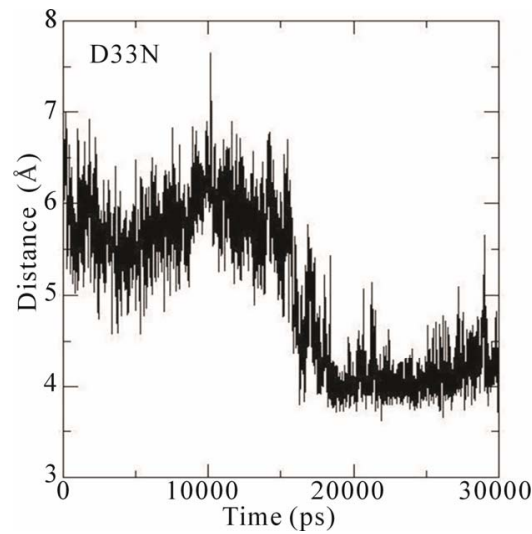

(c)

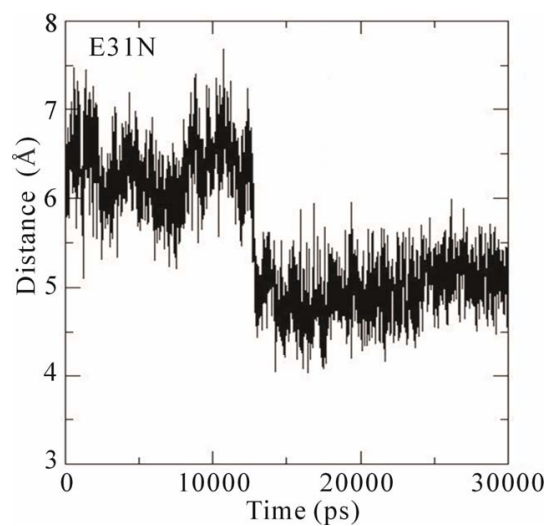

(b)

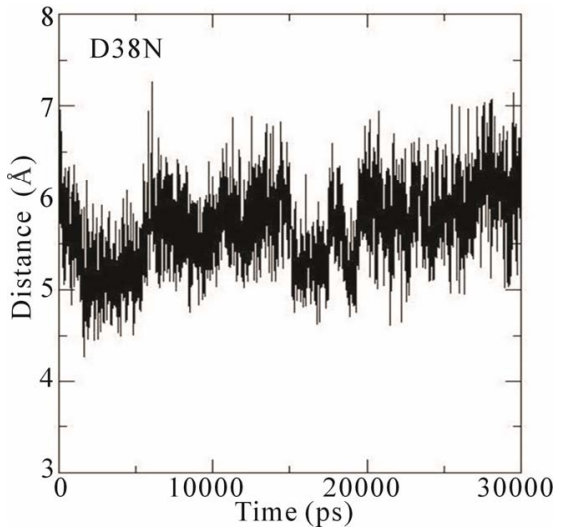

(d)

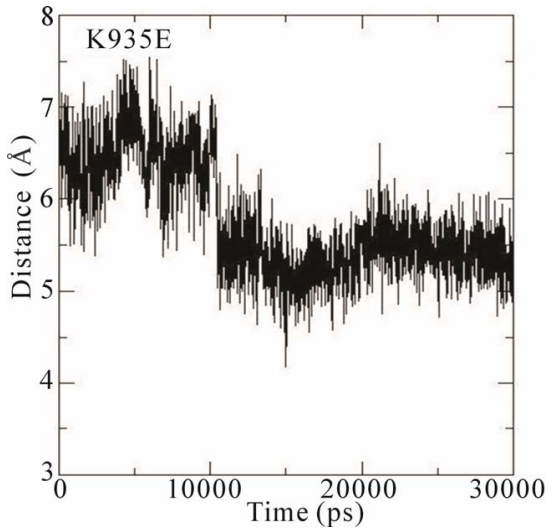

(e)

Figure 5. Distance (in $\AA$ ) between the side chain phenol ring of Tyr32 and the guanidinium group of Arginine789 of the wildtype and mutant systems, over $30 \mathrm{~ns}$ MD simulation.

K-Ras.GTP.GAP-334 and the E31N-Ras.GTP.GAP-334, D33N-Ras.GTP.GAP-334, D38N-Ras.GTP.GAP-334 and K-Ras.GTP.K935E-GAP-334 mutants, using molecular mechanics based methods. We find that the mutations affect the interaction of K-Ras and GAP by causing unfavorable conformations of the Switch I region and leaving an incompletely open Tyr32 gate, which in turn hinders the interaction of the catalytically critical residue Arg789 with GTP. This study enables us to better understand the precise changes in the protein structures that are brought out by mutated residues other than the hot spot codons, and how this leads to failed protein-protein interaction and oncogenic effects.

\section{Acknowledgements}

The Faculty of Science at the University of Gothenburg is gratefully acknowledged for financial support. We also acknowledge grants of computing time by the Swedish national infrastructure committee (SNIC).

\section{REFERENCES}

[1] K. Wennerberg, K. L. Rossman and C. J. Der, "The Ras
Superfamily at a Glance," Journal of Cell Science, Vol. 118, No. 5, 2005, pp. 843-846. doi:10.1242/jcs.01660

[2] M. Macaluso, G. Russo, C. Cinti, et al., "Ras Family Genes: An Interesting Link between Cell Cycle and Cancer," Journal of Cellular Physiology, Vol. 192, No. 2, 2002, pp. 125-130. doi:10.1002/jcp.10109

[3] A. Fernández-Medarde and E. Santos, "Ras in Cancer and Developmental Diseases," Genes \& Cancer, Vol. 2, No. 3, 2011, pp. 344-358. doi:10.1177/1947601911411084

[4] J. L. Bos, "Ras Oncogenes in Human Cancer: A Review," Cancer Research, Vol. 49, No. 17, 1989, pp. 4682-4689.

[5] C. A. Ellis and G. Clark, "The Importance of Being KRas," Cellular Signalling, Vol. 12, No. 7, 2000, pp. 425434. doi:10.1016/S0898-6568(00)00084-X

[6] J. W. Keller, J. L. Franklin, R. Graves-Deal, et al., "Oncogenic KRAS Provides a Uniquely Powerful and Variable Oncogenic Contribution among RAS Family Members in the Colonic Epithelium," Journal of Cellular Physiology, Vol. 210, No. 3, 2007, pp. 740-749. doi:10.1002/jcp.20898

[7] I. Macara, K. Lounsbury, S. Richards, et al., "The Ras Superfamily of GTPases," The FASEB Journal, Vol. 10, No. 5, 1996, pp. 625-630.

[8] K. Scheffzek, M. R. Ahmadian, W. Kabsch, et al., "The Ras-RasGAP Complex: Structural Basis for GTPase Activation and Its Loss in Oncogenic Ras Mutants," Science, 
Vol. 277, No. 5324, 1997, pp. 333-339. doi:10.1126/science.277.5324.333

[9] C. Kötting, A. Kallenbach, Y. Suveyzdis, et al., "The GAP Arginine Finger Movement into the Catalytic Site of Ras Increases the Activation Entropy," Proceedings of the National Academy of Sciences, Vol. 105, No. 17, 2008, pp. 6260-6265. doi:10.1073/pnas.0712095105

[10] H. te Heesen, K. Gerwert and J. Schlitter, "Role of the Arginine Finger in Ras RasGAP Revealed by QM/MM Calculations," FEBS Letters, Vol. 581, No. 29, 2007, pp. 5677-5684. doi:10.1016/j.febslet.2007.11.026

[11] B. L. Grigorenko, A. V. Nemukhin, I. A. Topol, et al., "QM/MM Modeling the Ras-GAP Catalyzed Hydrolysis of Guanosine Triphosphate," Proteins: Structure, Function, and Bioinformatics, Vol. 60, No. 3, 2005, pp. 495-503. doi:10.1002/prot.20472

[12] H. Kiaris and D. A. Spandidos, "Mutations of Ras Genes in Human Tumours (Review)," International Journal of Oncology, Vol. 7, No. 3, 1995, pp. 413-421.

[13] P. Wegman, C. Ahlin and B. Sorbe, "Genetic Alterations in the K-Ras Gene Influence the Prognosis in Patients with Cervical Cancer Treated by Radiotherapy," International Journal of Gynecological Cancer, Vol. 21, No. 1, 2011, pp. 86-91. doi:10.1097/IGC.0b013e3182049924

[14] S. R. Lin, C. H. Hsu, J. H. Tsai, et al., "Decreased GTPase Activity of K-Ras Mutants Deriving from Human Functional Adrenocortical Tumours," British Journal of Cancer, Vol. 82, No. 5, 2000, pp. 1035-1040.

[15] M. S. Nur-E-Kamal, A. Sizeland, G. D’Abaco, et al., "Asparagine 26, Glutamic Acid 31, Valine 45, and Tyrosine 64 of Ras Proteins Are Required for Their Oncogenicity," Journal of Biological Chemistry, Vol. 267, No. 3, 1992, pp. 1415-1418.

[16] C. Oliveira, J. L. Westra, D. Arango, et al., "Distinct Patterns of KRAS Mutations in Colorectal Carcinomas According to Germline Mismatch Repair Defects and hMLH1 Methylation Status," Human Molecular Genetics, Vol. 13, No. 19, 2004, pp. 2303-2311. doi: $10.1093 / \mathrm{hmg} / \mathrm{ddh} 238$

[17] Y. Li, G. Bollag, R. Clark, et al., "Somatic Mutations in the Neurofibromatosis 1 Gene in Human Tumors," Cell, Vol. 69, No. 2, 1992, pp. 275-28. doi:10.1016/0092-8674(92)90408-5

[18] K. Scheffzek, M. R. Ahmadian, L. Wiesmüller, et al., "Structural Analysis of the GAP-Related Domain from Neurofibromin and Its Implications," The EMBO Journal, Vol. 17, No. 15, 1998, pp. 4313-4327. doi:10.1093/emboj/17.15.4313

[19] Molecular Operating Enviroment (MOE), Chemical Computing Group: Montreal, 2009.
[20] B. Hess, C. Kutzner, D. van der Spoel, et al., "GROMACS 4: Algorithms for Highly Efficient, Load-Balanced, and Scalable Molecular Simulation," Journal of Chemical Theory and Computation, Vol. 4, No. 3, 2008, pp. 435447. doi:10.1021/ct700301q

[21] J. Wang, P. Cieplak and P. A. Kollman, "How Well Does a Restrained Electrostatic Potential (RESP) Model Perform in Calculating Conformational Energies of Organic and Biological Molecules?" Journal of Computational Chemistry, Vol. 21, No. 12, 2000, pp. 1049-1074. doi:10.1002/1096-987X(200009)21:12<1049::AID-JCC3 $>3.0 . \mathrm{CO} ; 2-\mathrm{F}$

[22] K. L. Meagher, L. T. Redman and H. A. Carlson, "Development of Polyphosphate Parameters for Use with the AMBER Force Field," Journal of Computational Chemistry, Vol. 24, No. 9, 2003, pp. 1016-1025. doi:10.1002/jcc. 10262

[23] U. Essmann, L. Perera, M. Berkowitz, et al., "A Smooth Particle Mesh Ewald Method," The Journal of Chemical Physics, Vol. 103, No. 19, 1995, pp. 8577-8593. doi:10.1063/1.470117

[24] T. Darden, D. York and L. Pedersen, "Particle Mesh Ewald: An $N \log (\mathrm{N})$ Method for Ewald Sums in Large Systems," The Journal of Chemical Physics, Vol. 98, No. 12, 1993, pp. 10089-10092. doi:10.1063/1.464397

[25] H. J. C. Berendsen, J. P. M. Postma, W. F. van Gunsteren, et al., "Molecular Dynamics with Coupling to an External Bath," The Journal of Chemical Physics, Vol. 81, No. 8, 1984, pp. 3684-3690. doi:10.1063/1.448118

[26] B. Hess, H. Bekker, H. J. C. Berendsen, et al., "LINCS: A Linear Constraint Solver for Molecular Simulations," Journal of Computational Chemistry, Vol. 18, No. 12, 1997, pp. 1463-1472. doi:10.1002/(SICI)1096-987X(199709)18:12<1463::AID -JCC4>3.0.CO;2-H

[27] W. G. Hoover, "Canonical Dynamics: Equilibrium PhaseSpace Distributions," Physical Review A, Vol. 31, No. 3, 1985, pp. 1695-1697. doi:10.1103/PhysRevA.31.1695

[28] M. Parrinello and A. Rahman, "Polymorphic Transitions in Single Crystals: A New Molecular Dynamics Method," Journal of Applied Physics, Vol. 52, No. 12, 1981, pp. 7182-7190. doi:10.1063/1.328693

[29] A. A. Gorfe, B. J. Grant and J. A. McCammon, "Mapping the Nucleotide and Isoform-Dependent Structural and Dynamical Features of Ras Proteins," Structure (London, England: 1993), Vol. 16, No. 6, 2008, pp. 885-896.

[30] S. Lukman, B. J. Grant, A. A. Gorfe, et al., "The Distinct Conformational Dynamics of K-Ras and H-Ras A59G," PLoS Computational Biology, Vol. 6, No. 9, 2010, Article ID: e1000922. doi:10.1371/journal.pcbi.1000922 


\section{Supplement}

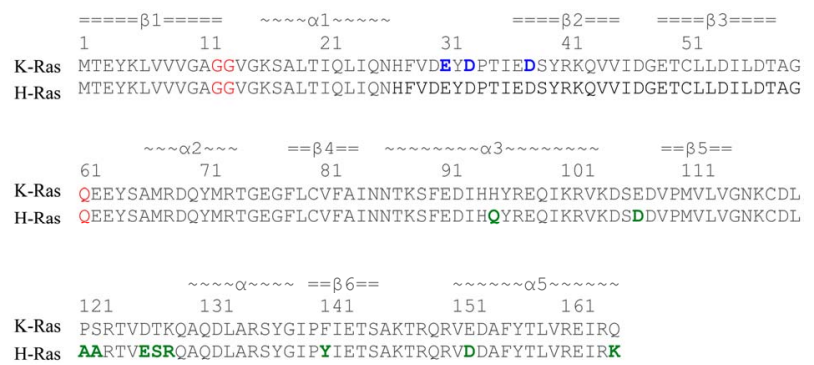

(a)

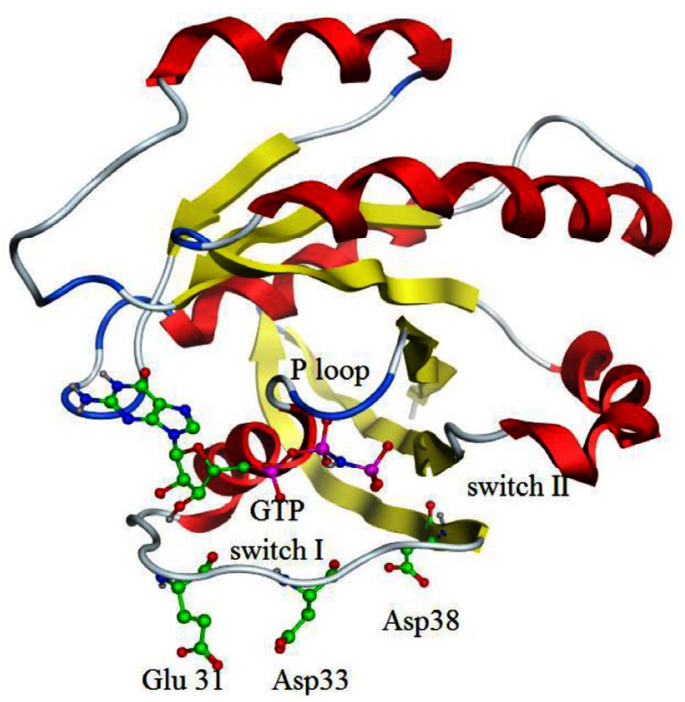

(b)

Figure S1. Primary and tertiary structure of K-Ras. (a) The sequence of $K-$ Ras and $H$-Ras, with amino acid differences indicated in green. Residues 12, 13, and 61, whose substitutions are associated with a large number of cancers, are colored red. Residues studied in this paper, where mutations have been associated with various cancers are colored in blue. Secondary structure content is indicated at the top of the sequences; (b) The Ras catalytic domain. P-loop (residues 10 - 17), Switch I (30 - 40) and Switch II (60 - 70) region are indicated, as well as the mutant residues Glu31, Asp33 and Asp38.

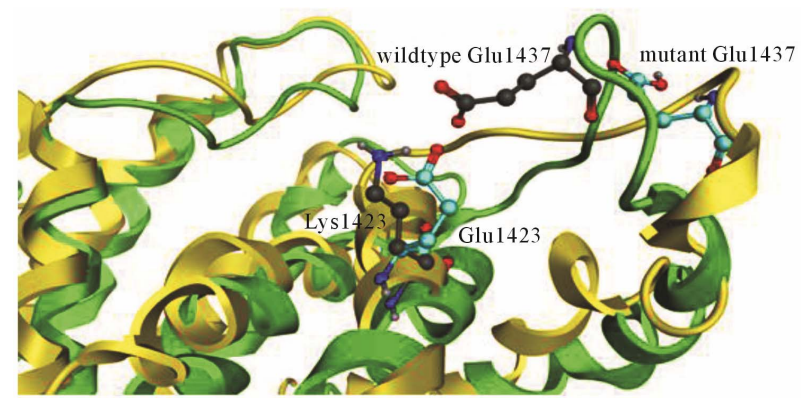

Figure S2. Structure alignment of wildtype and mutant K1423E NF1-333 GAP. The backbone of the wildtype is colored in green and the mutant in yellow, the carbon atoms of residues in wildtypecolored in grey and mutant in light blue.

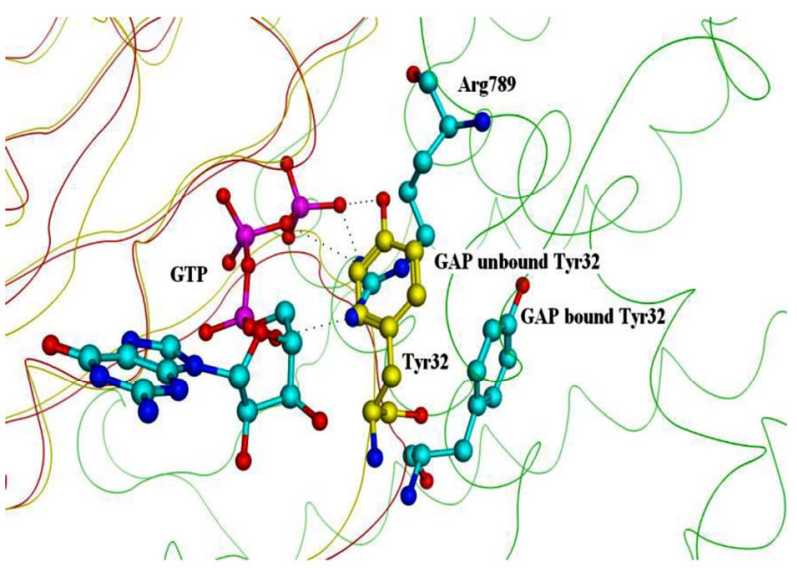

Figure S3. Conformation of Residue 32. Superposition of GAP-bound and unbound Ras. Tyr32 in unbound Ras (yellow) clashes with Arg789 of GAP.

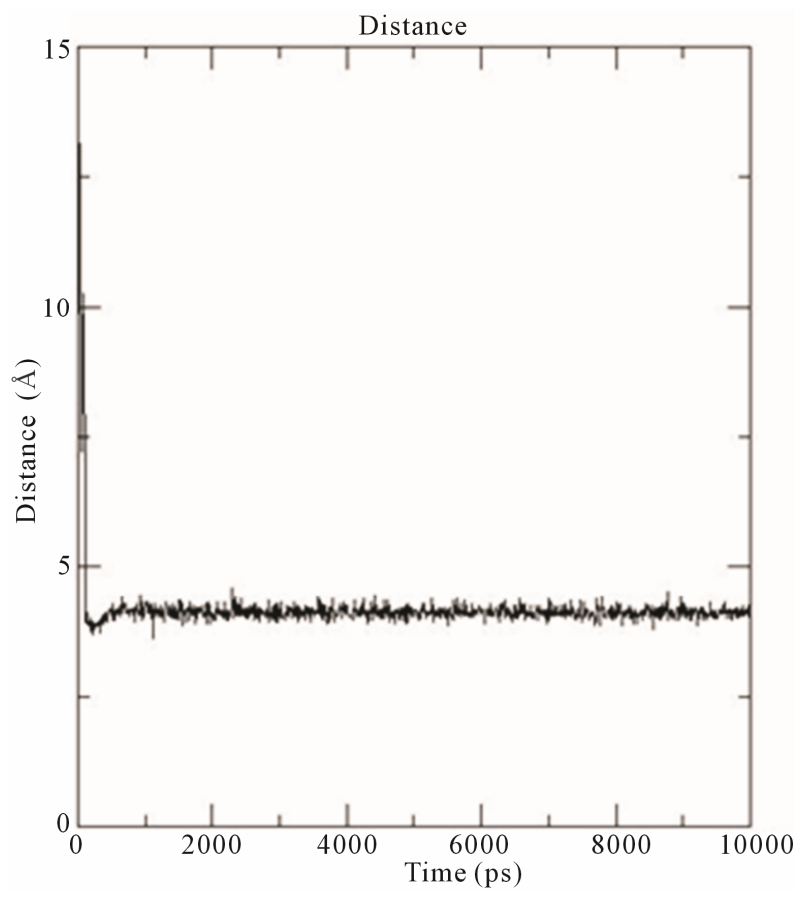

Figure S4. Distance between the O-H group of the Tyr32 side chain and the $\gamma$ phosphate group of GTP during the simulation. The GAP-unbound K-Ras with open Tyr32 gate was isolated from the K-Ras.GTP.GAP complex and a 100 ns MD simulation performed (parameters as described in the Materials and Methods) without water due to computational limitations. Already from the beginning of the simulation, the Tyr32 gate in isolated K-Rasmoves back to contact the $\gamma$ phosphate group, as seen from the distance between the O-H group of the Tyr32 side chain and the $\gamma$ phosphate group of GTP which reduces from 13 to $4 \AA$. 